\title{
Caracterización de las prácticas contables de costos y presupuestos en las unidades productivas de la comuna 8 de Villavicencio
}

\section{Characterization of accounting practices of costs and budgets in the productive units of the commune 8 of Villavicencio}

Julieth-Lorena Velásquez-Carrillo

Artículo de investigación:

Fecha de recepción:

2019/11/20

Fecha de aceptación:

2019/12/16

Esta publicación se encuentra bajo licencia:

Creative Commons

Reconocimiento-

NoComercial-

SinObraDerivada 4.0

Internacional

\section{Resumen}

Este artículo presenta los resultados del proyecto de investigación sobre la caracterización de las prácticas contables de costos y presupuestos de las unidades productivas de la comuna 8 de Villavicencio en el barrio de Ciudad Porfía. El estudio se efectuó por medio de técnicas como la observación, entrevistas y encuestas que permitieron caracterizar y evaluar la gestión del costo en setenta y tres (73) empresas y mostrar las principales características del proceso de gestión del costo, proceso de planeación, presupuesto de gastos, control y toma de decisiones. La ausencia de sistemas de costos, estructuración de un presupuesto de gastos, análisis de información contable para la toma de decisiones, que conllevan al cierre de los establecimientos, resultaron ser las variables más comunes en este tipo de negocios, así como la informalidad de sus actividades comerciales y de la contratación de sus trabajadores.

Palabras claves: Contabilidad, Presupuesto, Costos, Mantenimiento de registros.

Economista, Especialista en Gestión de la calidad, Maestrante en Dirección y Gestión de Proyectos, Universidad Santo Tomás, Colombia, Velasquezc. julieth@gmail.com, código ORCID: https://orcid.org/0000-0001-9386-0728 


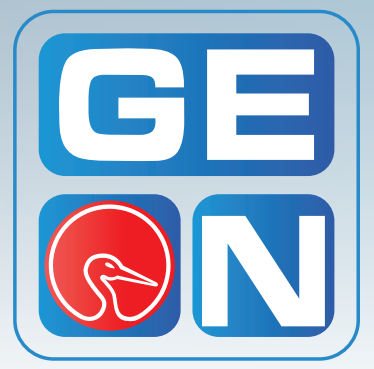

\section{Revista GEON}

(Gestión, Organizaciones y Negocios)

ISSN: 2346-3910 en línea

revistageon@unillanos.edu.co

Universidad de los Llanos

Colombia

\section{Velasquez Carrillo, J. (2020).}

Caracterización de las prácticas contables de costos y presupuestos en las unidades productivas de la comuna 8 de Villavicencio.

\section{Revista GEON}

(Gestión, Organizaciones y Negocios), 7(1), 165-178.

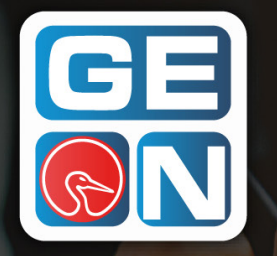

Revista GEON

$f$ in $\theta$

66 ...ausencia de sistemas de costos,

estructuración de un presupuesto de gastos, análisis de información contable para la toma de decisiones, que conllevan al cierre de los establecimientos 99

Velasquez Carrillo, J. (2020). Caracterización de las prácticas contables de

costos y presupuestos en las unidades productivas de la comuna 8 de

Villavicencio. Revista GEON (Gestión, Organizaciones Y Negocios), 7(1).

\section{http://revistageon.unillanos.edu.co}




\begin{abstract}
This article show the result of the investigation project about the identification of accounting practices of cost and the budgets of the productive units in the Villavicencio Commune 8, Ciudad Porfía neighborhood. The study was executed trough of techniques as observation, interviews and surveys that allowed identify and assess the cost management in seventy-three (73) entreprises and evince the main characteristics of the cost management process, planning process, expense budget, control and take decisions. The absence of cost systems, conformation of expenses budget, accounting information analysis in order to take decision result in the close of the establishments. these variables result the factors more comon in these kind of business as well as the informality of their commercial activities and the workers hiring
\end{abstract}

Keywords: Accounting, Budgeting, Cost, Record Keeping.

Códigos JEL: M41

\section{Introducción}

Villavicencio es la capital del departamento del Meta, y es el centro comercial más importante de los Llanos Orientales. Es el municipio más poblado del departamento del Meta seguido por Acacias y Granada, cuenta con una amplia oferta comercial e institucional, lo que lo convierte en un municipio propicio para la inversión y desarrollo económico, social, de infraestructura e institucional (Cámara de Comercio de Villavicencio, 2017).

El desarrollo económico de la ciudad ha permitido la generación de nuevas microempresas a lo largo del casco urbano. La facilidad en la creación de este tipo de empresas, donde no se requiere mayor capital para su inversión, así como la poca especialización que se demanda para el funcionamiento de la misma, proporcionan las condiciones socioeconómicas para la aparición activa de nuevos comercios de este arquetipo.

Acerca de su relevancia y aporte a la economía, la CEPAL argumenta que las mipymes "representan actores claves para incrementar el crecimiento potencial de América Latina. Estas empresas se caracterizan por una gran heterogeneidad en su acceso a mercados, tecnologías y capital hu- 
mano, así como su vinculación con otras empresas, factores que afectan su productividad, capacidad de exportación y potencial de crecimiento" (CEPAL, 2019).

A su vez, se ha dicho que las pequeñas empresas "constituyen un componente fundamental del entramado productivo en la región: representan alrededor de 99\% del total de empresas y dan empleo a cerca de $67 \%$ del total de trabajadores" (CEPAL, 2019), por ende, son un agente económico relevante para la economía de cualquier país o región. Es por ello que se dirigen variados esfuerzos al fomento y apoyo de este tipo empresarial en áreas como financiamiento, capacitación, formalización, desarrollo competitivo y estímulos tributarios, puesto que la pyme en Latinoamérica desempeña un papel de importancia en el desarrollo económico de los países y en la generación de empleo (Banco Interamericano para el Desarrollo, 2000)

Para esta investigación particular, en la comuna 8 de la ciudad de Villavicencio se ha evidenciado un activo desarrollo urbano y crecimiento de este tipo de pequeñas empresas. Son unidades productivas que realizan variadas actividades económicas de manera independiente en el sector comercial, de servicios y de manufactura, aportando a la generación de empleo.

Sin embargo, de la manera como estas se constituyen y operan, bajo conocimientos empíricos y rudimentarios, no propios de una organización formal con una cultura poco arraigada hacia el control de la gestión y la toma de decisiones basada en hechos, limitan las posibilidades de llegar a estados de longevidad, reconocimiento y expansión en los mercados.

Si bien el aporte que hacen las microempresas a la economía es indiscutible por su representativa participación, en muchos casos no existen las condiciones y entornos, tanto internos como externos, que favorezcan su competitividad y supervivencia en los mercados ante épocas de desaceleración económica, dificultando ser el motor que podrían ser para el desarrollo socioeconómico de cualquier región.

Por ello, es sustancial analizar los factores internos que afecten sus potencialidades, especialmente aquellas enfocadas a su gestión contable para la toma de decisiones estratégicas, de manera que estas puedan llegar a ser rentables y sostenibles a través del tiempo. Por tanto, se hace oportuno el análisis de las estructuras contables de las unidades productivas de la comuna 8 que recientemente están emergiendo en este mercado local, identificar sus fortalezas y falencias y brindar las herramientas para que los empresarios puedan optimizar su estructura de costos, a fin de obtener los resultados particulares esperados, la sostenibilidad de empleo y el desarrollo comercial, social y económico de esta población.

Como objetivo principal del estudio se planteó caracterizar las prácticas contables de costos y presupuestos de las unidades productivas de la comuna 8 de Villavicencio, cuya pregunta problémica se centró en: 
¿Cuáles son las características de las prácticas contables sobre los costos y presupuestos en las unidades productivas de la comuna 8 de Villavicencio?

\section{Contexto teórico}

El concepto de costos y presupuestos aborda diferentes dimensiones y significados. Según Edward Menesby "el costo se define como la medición en términos monetarios, de la cantidad de recursos usados para algún propósito u objetivo, tal como un producto comercial ofrecido para la venta general o un proyecto de construcción. Los recursos emplean materia prima, materiales de empaque. Horas de mano de obra trabajada, prestaciones, personal salariado de apoyo, suministros y servicios comprados y capital atado en inventario, terrenos edificios y equipo" (Menesby, 2015).

Mallo y Kaplan refieren versiones de costo desde diferentes áreas: "...el concepto económico del costo se ha utilizado en dos versiones generales: la primera, en sentido de consumo o sacrificio de recursos de factores productivos, y la segunda en el sentido de costo alternativo o de oportunidad" (Mallo, 2000)

Harry Howe, define al costo como el "precio pagado o la retribución para adquirir un activo. Aplicando a los inventarios, el costo significa en principio la suma de los desembolsos aplicables y cargos incurridos directa o indirectamente al traer un artículo a su condición y localización existente".

Baker y Jacobsen definen el costo "... como una suma de erogaciones, es decir, el costo inicial de un activo o servicio adquirido se refleja en el desembolso de dinero en efectivo y otros valores, o sea un pasivo incurrido" (Baker, 1988).

Puede entonces llegar a una aproximación de la definición del costo al indicar que este es el desembolso económico y no económico que implica la oferta de un bien o servicio durante su proceso productivo y/o de venta.

De otra parte, su clasificación por su comportamiento o incidencia, puede desagregarse en costo variable y costo fijo. Los costos fijos no cambian en función de los niveles de producción, mientras que los costos variables si se modifican de manera correlativa con la cantidad de bienes generados o servicios prestados.

En relación a un sistema de costos, se dice que:

Un sistema de costos es un conjunto de procedimientos, técnicos, administrativos y contables que se emplea en un ente, para determinar el costo de sus operaciones en sus diversas fases, de manera de utilizarlo para fines de información contable, control de gestión y base para la toma de decisiones. (Gerencie, 2017).

Es necesario tener en cuenta que:

La determinación de costos es un parte importante para lograr el éxito en cualquier negocio. Con ella podemos conocer a tiempo si el precio al que vendemos lo que producimos nos permite lograr la obtención de beneficios, luego de 
cubrir todos los costos de funcionamiento de la empresa. (Gonzales \& Serpa, 2008)

La palabra presupuesto se compone de dos palabras latinas: PRE = que significa "antes de" y SUPUES$\mathrm{TO}=$ indica "hecho, formado", por consiguiente, presupuesto significa "Antes de hecho" (Del Rio, 2009).

Al igual que la definición de costo, la definición de presupuesto está dado por diversos autores y disciplinas.

Klosowski, plantea que el presupuesto es una hoja de trabajo, que describe su estilo de vida (o tipo de vida). Un presupuesto, es para su programa de equilibrio financiero, lo que una dieta es, para su programa de equilibrio físico. El objetivo primordial de un presupuesto, es mejorar su situación en un futuro, con un sistema de gasto disciplinado (Klosowski, 1991)

El presupuesto es una herramienta que le permite saber cuáles son sus ingresos y gastos en un tiempo determinado, conocer cuánto puede destinar al ahorro para el cumplimiento de sus metas planteadas, identificar en qué está gastando su dinero, cuánto necesita para cubrir sus necesidades, determinar en qué está gastando de más y tomar medidas cuando se requiera hacer un recorte de gastos. (Asobancaria, 2019).

Mendoza, abrevia la definición de presupuesto como un completo plan financiero diseñado para orientar al empresario hacia la consecución de las metas propuestas (Mendoza, 2004).
Coincidiendo con lo afirmación de Ramírez: "En términos generales, se puede decir, que un presupuesto es una herramienta de planificación, coordinación y control de funciones que presenta en términos cuantitativos las actividades que en el futuro se realizarán en una empresa, con la finalidad de utilizar de una manera más productiva sus recursos, para que pueda alcanzar sus metas. Es un plan de acción encaminado al cumplimiento de una meta, la cual debe expresarse en términos de valores y financieros y cumplirse en un tiempo determinado, bajo ciertas condiciones estipuladas" (Ramirez, 2011).

Así como lo afirma Ortiz: "cada vez que se inicia un proceso de reestructuración financiera, el presupuesto asume su primer objetivo de planeación; en donde de manera sistemática se inician un conjunto de acciones enfocadas a escenificar lo que pudiera suceder en el futuro (suponer) $y$, en función de ello, establecer las estrategias necesarias para anticiparse (pre) a él. Por lo que elaborar un presupuesto no es matemática cuántica ni física espacial. Es solo avocarse a visualizar escenarios posibles que permitan la creación de alternativas viables. (Ortiz, 2014)

\section{Materiales y métodos}

La investigación se desarrolló a partir de un trabajo de campo de tipo descriptivo, considerando llegar a conocer las prácticas de costos y presupuestos de las unidades productivas de la comuna 8 de Villavicencio. Ostenta un enfoque mixto, por tratar- 
se según Sampieri de "un conjunto de procesos sistemáticos, empíricos y críticos de investigación e implican la recolección y el análisis de datos cuantitativos y cualitativos, así como su integración y discusión conjunta, para realizar inferencias producto de toda la información recabada (metainferencias) y lograr un mayor entendimiento del fenómeno bajo estudio" (Sampieri, 2014)

En la primera fase se estructuró un instrumento de encuesta para la recolección de datos acerca de las prácticas de costos y presupuestos de las unidades productivas de la comuna 8. La herramienta contenía diferentes preguntas de selección múltiple de única respuesta y otras con una oportunidad de respuesta abierta con diferentes tipos de preguntas orientadas a: contextualizar y caracterizar las prácticas comerciales y contables de la empresa, identificar los costos fijos y variables, ingresos y utilidades, así como otros aspectos del comercio.

El instrumento fue sometido a una prueba piloto con unidades productivas de la comuna 8 de Villavicencio, cuyos resultados permitieron afianzar la herramienta para su aplicación in situ. Por su parte, el universo comprendió un total de 299 unidades productivas, censadas en la localidad del barrio Ciudad Porfía, en la zona geográfica limitada sobre la carrera 43 entre las calles 53 sur hasta la caIle 70 sur, selecto por considerarse como uno de los barrios más extensos y poblados de la comuna 8, y la carrera 43 por ser la vía principal del sector y una de las de mayor concentración de unidades productivas.
En cuanto a la muestra, esta fue de 56 empresas, en función a un muestreo aleatorio simple, con una confiabilidad del $90 \%$ y margen de error del 10\%, sin embargo, se llevó la aplicación del instrumento a un total de 73 unidades productivas.

En la segunda fase, se realizó la interpretación y análisis de los resultados, con el uso de la herramienta Excel en función de los objetivos trazados y a través de uso de fuentes de información primarias y secundarias se efectuó una triangulación de los datos para dar respuesta a la pregunta problémica planteada para el presente estudio.

\section{Resultados}

La encuesta fue atendida en su gran mayoría por empleados que se encontraban en las unidades productivas (66\%), el 19\% de las respuestas provinieron de los administradores de los negocios y el restante (15\%) de los dueños. A razón de la caracterización anterior, muchas respuestas del manejo contable eran desconocidas por los empleados, aislando parcialmente el alcance final de la información esperada.

La identificación y contexto de las unidades productivas permitió reconocer que el 100\% de los encuestados son empresas pertenecientes al sector comercial, con una caracterización genérica de menos de 10 trabajadores e ingresos anuales inferiores a 44.769 UVT, clasificándolas como microempresas, según el Decreto 957 de 2019 (Ministerio de Comercio, 2019). 
Al respecto de su formalización, el $81 \%$ aseguró contar con registro mercantil, de estos el 52\% se encuentra registrado como establecimiento comercial; tan sólo el 9.6\% no cuenta con el reconocimiento ante la Cámara de Comercio. Sin embargo, es relevante destacar que sólo el 22\% de las 59 empresas que poseen registro mercantil han efectuado la renovación del mismo durante la vigencia 2019. Ninguno de los encuestados ubicaba en un lugar visible su registro mercantil.

El mismo patrón de formalización de las unidades productivas se repite al interrogar si cuenta con registro único tributario-RUT, donde 52\% afirma tenerlo, $12.3 \%$ no lo tiene y $35.7 \%$ desconoce si cuenta con RUT. Teniendo en cuenta la Ley 1943 de 2018 en materia de descuento del IVA de los bienes de capital y el nuevo esquema simple es indicado que los empresarios deben solicitar el RUT de manera más ágil con el sistema electrónico, la situación anterior parece atribuirse a un desconocimiento por parte de los comerciantes ante sus obligaciones.

Sobre la contratación de los empleados, un porcentaje significativo (82\%) manifestó realizar una contratación de tipo verbal con sus trabajadores, con características de subordinación y cumplimiento de horarios. El 89\% de los encuestados no realiza pago de aportes parafiscales por parte del empleador o del trabajador.

En cuanto al registro de la actividad contable del establecimiento, el 44\% lleva un registro de sus operaciones en cuadernos o libretas, sin la aplica- ción de una metodología contable o el uso de los principios generalmente aceptados en contabilidad, 5\% utiliza softwares contables, $7 \%$ en documentos de Excel, 11\% en libros columnarios, $14 \%$ lo hace a través de un contador externo y 19\% no lleva registro contable de ningún tipo. Las razones atribuidas por las cuales argumentaron no se lleva ningún tipo de contabilidad, son que no la consideran útil (50\%) o no saben cómo ejecutarla (50\%). De lo anterior, puede inferirse que un porcentaje significativo de los negocios no ejecuta la contabilidad de acuerdo a las prescripciones legales, presumiéndose que optan por una contabilidad menos técnica, y más rudimentaria, con más falencias, y menos garantías, lo que dificultaría la toma de decisiones, control interno y control fiscal sobre las actividades comerciales.

Sobre las proyecciones de ventas de su establecimiento, 40 unidades productivas (55\%) no programan metas en largo ni corto plazo, el 42\% las planifica en un corto plazo de 30 días y 3\% a 12 meses, como puede observarse en la figura 1.

Figura 1. Proyección de ventas de la unidad productiva

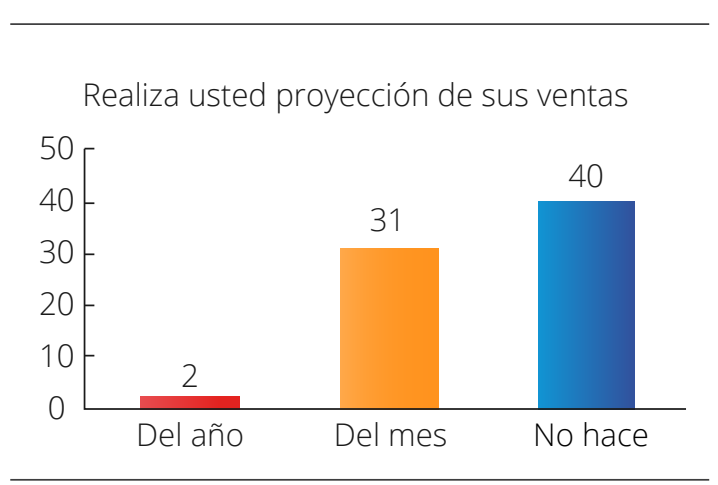

Fuente: Encuestas aplicadas en las unidades productivas de la comuna 8, Ciudad Porfía 
De igual forma, en este tipo de unidades productivas se encontró que las proyecciones de las compras de materias primas o productos para venta que se realizan, se basan en el stock que se tenga en el inventario (49.3\%) o de acuerdo al volumen de sus ventas (23\%) y un $26 \%$ deja la decisión de las compras a su intuición o experiencia en el negocio. El manejo de los inventarios es otra actividad que se realiza de forma rudimentaria, puesto que los documentos del control son en su mayoría hojas en libretas. Al entrevistar a los participantes sobre la trazabilidad de la información, se encontró que el $80 \%$ no puede identificar cuantas eran las unidades adquiridas y compradas en el mismo mes, pero del año inmediatamente anterior.

Los resultados anteriores evidencian una visión a muy corto plazo que tienen estos empresarios, demostrando muy poca planificación y control de su negocio lo que reduce sus posibilidades de crecimiento, y los hace más vulnerables ante épocas de recesión económica derivadas por factores del mercado local, regional o global.

Los gastos y costos de estas unidades en particular son registrados en un mayor porcentaje (40\%) con una periocidad diaria, el $14 \%$ se realiza semanalmente, $23 \%$ lo ejecuta mensualmente y el otro $23 \%$ no realiza ningún registro de sus gastos y costos o lo efectúa de manera ocasional.

La recolección de la información de los costos variables y fijos de estos establecimientos se dificultó puesto que no se tiene claridad con certeza de cuáles son los valores de los costos en los que incurre su establecimiento. De la misma manera, la información suministrada sobre los ingresos mensuales no fue coherente y apropiada, denotando una falta de confiabilidad de los encuestados en suministrar dicha información. La cultura de la informalidad de este tipo de unidades productivas lleva a considerar que el registro y análisis de la información contable por parte de asesores externos puede constreñir en sanciones legales o especulaciones sobre su proceder y no lo perciben como un apoyo a la gestión y toma de decisiones para su organización. A razón de lo anterior, no fue posible determinar puntos de equilibrio o utilidades que generan las empresas del estudio de la comuna 8.

El establecimiento de precios para los productos que se comercializa, se basa en mayor parte apoyado en el cálculo del porcentaje que se desee ganar con la venta del producto (44\%), un 30\% acoge los precios que los proveedores establecen y en menor escala (15\%) se calcula el precio de venta del producto por intuición o experiencia.

Lo anterior, incurre en que las empresas al momento de establecer los precios de venta de sus productos no tienen en cuenta la capacidad de adquisición permanente requerida sobre el nivel de ingresos suficientes para cumplir sus obligaciones de corto y largo plazo emanadas del ejercicio comercial y legal, de hecho, muchos de los propietarios encuestados aseguraron que no se asignan un salario para ellos mismos o efectúan declaraciones de renta sobre el monto que no es el real de sus ingresos. Esta puede ser una de las razones principales por las que estas unidades productivas no tengan 
un crecimiento y supervivencia en el largo plazo, obligándolas a cerrar en periodos muy cortos, pues los ingresos recibidos no son suficientes para el pago de las obligaciones.

Figura 2. ¿Cómo determina el precio de los productos que comercializa?

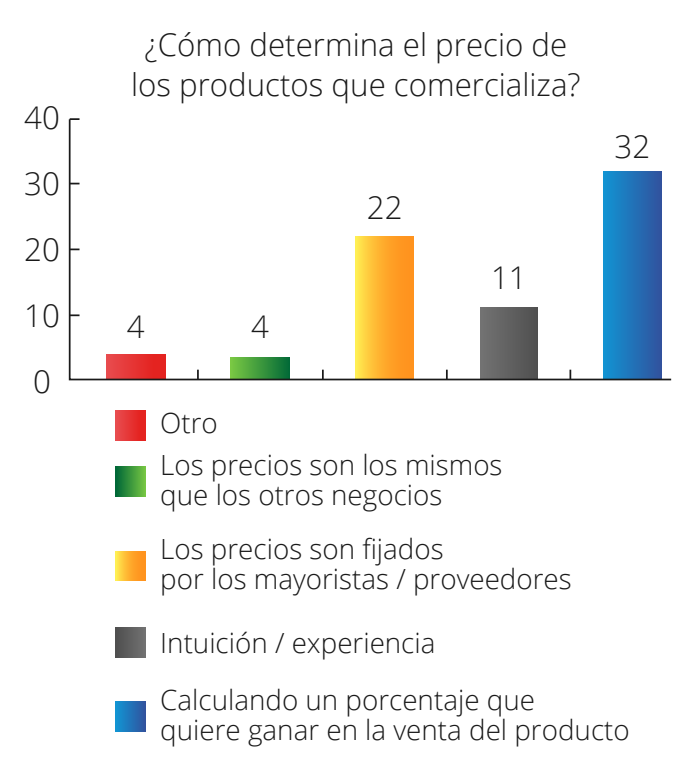

Fuente: Encuestas aplicadas en las unidades productivas de la comuna 8, Ciudad Porfía

En la mayoría de los casos los empresarios manifestaron no tener acceso a créditos del sistema financiero, limitando su capacidad de expansión y de inversión en nuevas tecnologías. Los créditos en los que incurrieron se han utilizado para cubrir su flujo de caja de corto plazo, en ninguno de los casos para inversión o expansión del propio negocio.

\section{Conclusiones}

La informalidad de la actividad comercial de las empresas que fueron parte del estudio fue una de las carac- terísticas generales encontradas. La legislación colombiana obliga a todo comerciante a realizar su registro mercantil y renovarlo en los tres primeros meses de cada año, así como llevar una contabilidad regular de sus negocios conforme a las prescripciones legales (Presidencia de la República, 1971), hecho que no se cumple de manera rigurosa en esta población.

Según los autores León y Caicedo en su investigación, este tipo de empresas "no están reguladas, tienen facilidad para entrar y salir del mercado debido a la poca exigencia de capital y calificación de mano de obra, y son generadoras de empleo familiar; en consecuencia, se convierten en una alternativa de supervivencia" (Camargo \& Caicedo, 2011).

En una definición dada por la CCV la informalidad se presenta cuando el micro establecimiento incumple cualquiera de los factores determinantes de informalidad, como son: no contar matrícula mercantil, no tener RUT, no tener afiliados a seguridad social a todos los trabajadores, no llevar contabilidad o no pagar impuestos (Cámara de Comercio de Villavicencio, 2018), afirmando la informalidad económica presente en el sector.

En correlación con lo anterior, los empresarios encuentados manifestron que no acudían a la formalización de sus negocios porque el costo de la formalización es alto y esto debido a que los esquemas normativos en el país, parecen acarrear más obligaciones tributarias y legales que beneficios para las empresas que se formalizan. Al respecto, multiples son las solucio- 
nes para disminuir esta informalidad, una de ellas está ligada a generar esquemas normativos favorables para la creación de empresas, tal y como lo precisa en su estudio los autores Pérez Paredes, Alfredo; Torralba Flores, Amado; Cruz de los Ángeles, José Aurelio. En su investigación argumentan que "cuando los emprendedores deciden crear una empresa, lamentablemente se encuentran con demasiados tramites y con un tiempo de repuesta demasiado lentos, esto desmotiva a lo potenciales empresarios y provoca que ya no quieren emprender o si lo hacen, optan por la informalidad" (Pérez Paredes, Torralba Flores, \& Cruz de los Ángeles, 2019).

Lo anterior requiere esfuerzos y voluntad de los diferentes órdenes de gobierno del país, de manera que se generen esquemas de apertura mucho más flexibles y rápidos para las nuevas empresas. En igual medida, generar políticas fiscales diferentes a las del orden de hoy en día, de modo que favorezcan e impulsen el crecimiento de las mismas promoviendo su formalización y desarrollo para aporte al PIB, tal y como lo propone en su misma investigación los autores Pérez Paredes, Alfredo; Torralba Flores, Amado; Cruz de los Ángeles, José Aurelio, la cual precisa: "Establecer regímenes fiscales atractivos para los emprendedores, abatirían uno de los problemas más fuertes que se tienen en el país y que es la informalidad que propicia la vasión de impuestos. Por ejemplo, el Régimen de Incorporación Fiscal (RIF) que actualmente existe en México, permite que las personas se den de alta y vayan pagando impuestos de forma paulatina hasta llegaral pago del 100\% de los impuestos después de diez años, lamentablemente no todos conocen este esquema y prefieren trabajar en la clandestinidad, si el gobierno se preocupará por este tema debiera fortalecer la transmisión de este tipo de información y generar las condiciones para que los emprendedores registren sus empresas a la Autoridad Fiscal" (Pérez Paredes, Torralba Flores, \& Cruz de los Ángeles, 2019)

Desde la perspectiva de los comerciantes del sector, las unidades productivas son creadas para su sostenimiento, pero no para su crecimiento o expansión, razón por la cual no mantienen una visión a largo plazo sobre el funcionamiento de sus comercios; esta aclaración fue precisada a través del dialogo con los encuestados donde las afirmaciones recurrentes entregadas fueron de "cerrar el negocio en caso de que este no de las utilidades esperadas", en un muy bajo porcentaje buscarían ayuda o asesoría externa y otros simplemente manifestaron no saber qué hacer ante una crisis.

Pese a que mayormente no se lleva una contabilidad de manera formal, mediante la visita, se identificó que se comprende un concepto empírico de lo que son derechos y obligaciones en términos económicos y aunque no hagan clasificación de los mismos sí tienen una percepción de qué y cuánto es de su propiedad y lo que corresponde a terceros. El uso de facturas también facilita la trazabilidad de la información sobre las actividades comerciales que se ejecutan en algunos casos.

Este patrón se asemeja a los resultados del estudio de Giraldo, Sines- 
terra y Arteaga realizado a las micro tiendas de la comuna 4 de Medellín en donde, "en la práctica contable concerniente a la clasificación la cual comprende la distribución de la cuantía de las transacciones económicas en categorías que bajo el estándar contable deberían cumplir con el criterio de partida doble, los tenderos no llevan registros económicos bajo estas características, pero por la misma consciencia del haber y deber descrita anteriormente se podría entender que guían su actuar y el manejo de los recursos bajo una racionalidad económica y contable" ( Giraldo Ruiz, Sinisterra Rincón, \& Arteaga Londoño, 2018)

Sin embargo, la evaluación de los costos no hace parte de las prácticas para la toma de decisiones internas y para el análisis de terceros, más aún, la estructuración de un presupuesto como herramienta para el cumplimiento de los objetivos del empresario no es una cuestión relevante dentro de las actividades de estas. Puede decirse entonces que, hay una carencia generalizada de las unidades productivas del barrio Ciudad Porfía de una planeación estratégica formal y continua en la gestión empresarial de sus mipymes. Lo anterior significa que las empresas se concentran en la operación del día a día o a lo sumo se tiene en cuenta un horizonte de corto plazo, generalmente de un año, donde la base fundamental de planeación son los presupuestos de ingresos y egresos (Zapata, 2004) y por ende no hay manera de que los empresarios perciban el efecto de las falencias de su empresa.

En este sentido, es de suma importancia fortalecer y adecuar las estruc- turas de costos y presupuestos de las unidades productivas de la comuna 8 de Villavicencio ya que esto permitirá que los empresarios de ésta localidad puedan implementar y/o potencializar sus sistemas de costos, optimizar los recursos, conocer su punto de equilibrio, incrementar su rentabilidad, realizar una planeación financiera óptima que le permita tener claridad sobre los recursos requeridos para su operación económica, las posibilidades de financiación y crecimiento a través del tiempo generando una consolidación que le permita la sostenibilidad en el largo plazo.

Como lo argumenta González "Los métodos y procedimientos que utilice la organización para la planificación, el registro de los gastos y el cálculo de los costos dependerán, en gran medida, del tipo de producción al que se dedique o el servicio que oferte, así como de la complejidad, el nivel de automatización, la relación costo-beneficio; en fin, de las características de la empresa" (Delgado, 2014)

Empero, más allá de los métodos que se recomienden implementar a los comerciantes del sector para la planificación, el registro y el cálculo del costo, debe otorgarse una especial atención a la cultura de administración de las unidades productivas, donde debe primar el criterio y la voluntad de utilizar el costo como un instrumento de dirección que permita a la administración de la entidad tomar las mejores decisiones y seleccionar aquella alternativa que brinde los mayores beneficios, con el mínimo de gastos. De igual forma, se debe lograr que se involucre a todos y cada 
uno de los trabajadores del negocio y adoptar técnicas y herramientas informáticas que permitan la trazabilidad de la información consultada.

Finalmente, la implementación de un presupuesto como hoja de ruta para el control de los gastos de las empresas, les permitirá direccionar, planear y controlar su negocio de manera eficiente, reconociendo que:

"El presupuesto es una herramienta muy valiosa para la empresa; en ella se planea integralmente todo lo referente a las operaciones que se llevan durante un periodo determinado. Su finalidad es auxiliar a la administración en el cumplimiento de los objetivos que se han propuesto los directivos" (Soto).

Como se ha mencionado con anterioridad, es necesario la educación financiera de los pequeños empresarios, y que los esfuerzos de las asociaciones comerciales de la ciudad, como de la cámara de comercio y universidades de la región, se centren en ofertar diversos portafolios para la capacitación del pequeño empresario, que incluya el fomentar una cultura del emprendimiento, la administración, la contabilidad y el control presupuestal como base del éxito para el crecimiento prolongado de sus pequeños negocios, de manera que con el tiempo se fortalezcan este tipo de estructuras económicas y lleguen a ser generadoras de empleo y de ingresos formales para la región.

\section{Referencias}

Giraldo Ruiz, C., Sinisterra Rincón, E., \& Arteaga Londoño, M. (2018). Prácticas contables generalmente utilizadas en el sector informal: Caracterización de las microtiendas de la comuna cuatro de Medellin. Contaduría Universidad de Antioquia 72(72), 59-80. doi:https:// doi.org/10.17533/udea.rc.n72a04

Asobancaria. (2018). ¿Por qué debemos implementar el programa de Educación Financiera en los colegios de Colombia? Obtenido de https:// www.asobancaria.com/wp-content/ uploads/1167.pdf

Baker, M. J. (1988). Contabilidad de costos. México: McGraw Hill Interamericana, Segunda edición.

Banco Interamericano para el DesarroIlo. (11 de 2000). Apoyo a la pequeña y mediana Empresa. New York: Publicaciones BID. Obtenido de Banco Interamericano para el Desarrollo : https://publications.iadb.org/es/ publicacion/16073/apoyo-la-pequena-y-mediana-empresa-una-decada-de-actividades-del-grupo-banco

Cámara de Comercio de Villavicencio. (Agosto de 2017). Análisis de los factores que afectan el desempeño económico del comercio en el centro de Villavicencio - Meta. Villavicencio: Cámara de Comercio de Villavicencio. Obtenido de Sector Comercio en VIllavicencio: http://www.ccv.org.co/site/fileadmin/ user_upload/Investigaciones_Economicas/SECTOR_COMERCIO_VILLAVICENCIO-revMGO_2_.pdf

Cámara de Comercio de Villavicencio. (2018). Censo de Establecimientos Informales de Villavicencio - Comuna 08. Obtenido de http://www.ccv.org.co/ site/fileadmin/user_upload/2017/Planeacion/Informe_C08_VF.pdf 
Camargo, A. L., \& Caicedo, H. G. (2011). La economía informal en Villavicencio. Finanzas y política económica, 83-103. Obtenido de https://revfinypolecon. ucatolica.edu.co/article/view/520

Castelló, E. (2018). El Pais. Obtenido de La educación financiera empieza en los centros escolares: https://cincodias. elpais.com/cincodias/2018/01/09/midinero/1515514953_731230.html

CEPAL, C. E. (2019). CEPAL. Obtenido de Acerca de microempresas y pymes: https://www.cepal.org/es/temas/pymes/acerca-microempresas-pymes

Del Rio, C. (2009). El presupuesto. México: Cenage Learning. Decima Edición .

Delgado, N. d. (2014). Estudio sobre la gestión del costo en las empresas cubanas. Cofin Habana.

Finanzas Para Todos. (s.f.). FinanzasParaTodos. Obtenido de Medios de pago: los cheques: http://finanzasparatodos.es/es/productosyservicios/productosbancariosoperativos/mediosdepagocheques.html

Gonzales, J. O. (2014). Finanzas y Proyectos. Obtenido de Importancia de la educación financiera: https:// finanzasyproyectos.net/importancia-de-la-educacion-financiera/

Hernández Sampieri, R. \&. (2008). El matrimonio cuantitativo cualitativo: el paradigma mixto.

Klosowski, A. (1991). Finanzas personales. México: Trillas. Primera Edición.

Mallo, C. K. (2000). Contabilidad de costos y estratégica de gestión. . Madrid: Prentice Hall.

Mendoza, C. (2004). Presupuestos para empresas de manufactura $\left(1^{a} \mathrm{Ed}\right.$. Barranquilla: Ediciones Uninorte.
Menesby, E. (2015). Costos y Presupuestos. Huancayo- Perú: Universidad Peruana los Andes.

Ministerio de Comercio, I. y. (5 de Octubre de 2019). Decreto 957 de 2019. Obtenido de https://www.accounter. co/wp-content/uploads/2019/06/ DECRETO-957-DEL-05-DE-JUNIODE-2019.pdf

Pérez Paredes, A., Torralba Flores, A., \& Cruz de los Ángeles, J. A. (2019). Propuestas para fortalecer la cultura emprendedora en los municipios de Puebla y Tlaxcala. Revista GEON (Gestión, Organizaciones Y Negocios), 6(2), 37-51. doi:https://doi.org/10.22579/2

Presidencia de la República. (1971). Código de Comercio. Obtenido de Decreto 410 de 1971: https://legislacion.vlex.com.co/vid/codigo-comercio-42856969\#section_6

Ramirez, C. V. (2011). Los presupuestos: sus objetivos e importancia. Revista Cultural Unilibre.

Sampieri, R. H. (30 de 10 de 2014). Metodología de la Investigación. México: Mc Graw Hill. Obtenido de http:// observatorio.epacartagena.gov. co/wp-content/uploads/2017/08/ metodologia-de-la-investigacionsexta-edicion.compressed.pdf

Soto, H. (s.f.). IEDGE Business School. Recuperado el 23 de Enero de 2019, de https://www.iedge.eu/homero-soto-la-importancia-del-presupuesto-dentro-de-la-empresa

Zapata, E. E. (2004). Las Pymes y su Problemática Empresarial: Análisis de caSOS. REVISTA ESCUELA DE ADMINISTRACIÓN DE NEGOCIOS No. 52, 118 - 135. 